\title{
Respiratory syncytial virus infection of newborn CX3CR1-deficient mice induces a pathogenic pulmonary innate immune response
}

\author{
Sudipta Das, ${ }^{1}$ Mahesh Raundhal, ${ }^{1}$ Jie Chen, ${ }^{1}$ Timothy B. Oriss, ${ }^{1}$ Rachael Huff, ${ }^{1}$ John V. Williams, ${ }^{2}$ \\ Anuradha Ray, ${ }^{1,3}$ and Prabir Ray ${ }^{1,3}$ \\ 'Division of Pulmonary, Allergy, and Critical Care Medicine, Department of Medicine, University of Pittsburgh School of \\ Medicine, Pittsburgh, Pennsylvania, USA. 'Division of Infectious Diseases, Department of Pediatrics, Children's Hospital \\ of Pittsburgh of University of Pittsburgh Medical Center, Pittsburgh, Pennsylvania, USA. ${ }^{3}$ Department of Immunology, \\ University of Pittsburgh School of Medicine, Pittsburgh, Pennsylvania, USA.
}

\begin{abstract}
Respiratory syncytial virus (RSV) infects almost all infants by 2 years of age, and severe bronchiolitis resulting from RSV infection is the primary cause of hospitalization in the first year of life. Among infants hospitalized due to RSV-induced bronchiolitis, those with a specific mutation in the chemokine receptor $\mathrm{CX} 3 \mathrm{CR} 1$, which severely compromises binding of its ligand $\mathrm{CX} 3 \mathrm{CL} 1$, were at a higher risk for more severe viral bronchiolitis than those without the mutation. Here, we show that RSV infection of newborn mice deficient in CX3CR1 leads to significantly greater neutrophilic inflammation in the lungs, accompanied by an increase in mucus production compared with that induced in WT mice. Analysis of innate and adaptive immune responses revealed an early increase in the number of IL-17+ $\gamma \delta$ T cells in CX3CR1-deficient mice that outnumbered IFN- $\gamma^{+} \gamma \delta \boldsymbol{T}$ cells as well as IFN- $\gamma^{+}$NK cells, IFN- $\gamma$ being host protective in the context of RSV infection. This bias toward

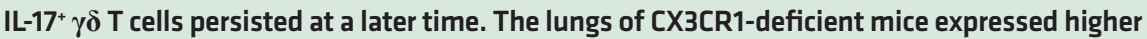
levels of IL-1 $\beta$ mRNA and protein, and blockade of IL-1 $\beta$ signaling using IL-1 receptor antagonist significantly reduced the number of $\mathrm{IL}-17^{+} \gamma \delta \mathrm{T}$ cells in the lungs of infected mice. Blockade of IL-17RC abolished RSV-induced lung pathology in infected CX3CR1-deficient mice. We propose that, in infants harboring mutant CX3CR1, targeting the IL-17R may minimize disease severity and hospitalization in early life.
\end{abstract}

Conflict of interest: The authors have declared that no conflict of interest exists.

Submitted: April 13, 2017 Accepted: July 25, 2017 Published: September 7, 2017

\section{Reference information:} JCI Insight. 2017;2(17):e94605. https://doi.org/10.1172/jci. insight.94605.

\section{Introduction}

Respiratory syncytial virus (RSV) is a common pathogen and the primary cause of hospitalization of infants in the first year of life (1-6). RSV infection is the leading cause of viral bronchiolitis in infants that can cause acute respiratory failure in pediatric intensive care units (2-7). In 2005, a global estimate of deaths caused by pneumonia in children $<5$ years old revealed RSV to be the third leading cause, accounting for close to 200,000 deaths (5). The virus only infects the respiratory mucosa and does not normally replicate outside of the bronchopulmonary tree. Unfortunately, no effective vaccine against RSV infection is yet available, and passive immunoprophylaxis using RSV-specific antibodies is expensive and only used in high-risk subjects $(8,9)$.

The major inflammatory response associated with RSV infection of infants with severe bronchiolitis includes neutrophil infiltration of the airways, epithelial sloughing, and mucus production. The virus has been found to infect both the proximal and distal airways as well as alveolar epithelial cells $(3,6,10)$. Interestingly, despite causing severe epithelial destruction in vivo, infection of airway epithelial cells (AECs) in culture does not cause cytopathology. This suggests a role for the inflammatory immune response triggered by virus infection in promoting epithelial damage and bronchiolitis.

RSV expresses 3 glycoproteins on its surface - F, G, and $\mathrm{SH}$. While the F protein is essential for fusion with the host cell membrane and infection, the $\mathrm{G}$ protein helps stabilize this interaction and has been associated with RSV-induced pathogenesis $(4,11,12)$. Complex formation between $\mathrm{F}$ and $\mathrm{G}$ proteins has been 
also suggested to enhance viral fusogenic activity. Studies using antibodies targeted against the G protein have shown an ability to modulate immune response against virus infection $(13,14)$. The $G$ glycoprotein can interact with the chemokine receptor CX3CR1 due to the presence of the CX3C motif in its amino acid sequence (15). The CX3C motif is present in the chemokine CX3CL1 (also known as fractalkine), which is the ligand of CX3CR1 (16). CX3CR1 expression on human AECs was shown to facilitate virus entry (17-19). However, deletion or knockdown of CX3CR1 expression in human AECs or in mice only partially reduces infection (19). It has been suggested that other molecules, such as glycosaminoglycans $(20,21)$ and annexin II (22), can also serve as binding partners for the $G$ protein on target cells. In an effort to understand RSV-induced severe bronchiolitis in infants, a previous study showed that infants carrying a specific $\mathrm{I}^{249} \mathrm{M}^{280}$ mutation in CX3CR1 experience more severe bronchiolitis in response to RSV infection than those without this mutation (23). This mutation in CX3CR1 attenuates binding of the receptor to its ligand CX3CL1 (fractalkine) (24). There is limited understanding of host-virus interactions that cause more severe illness in RSV-infected infants. Given the adverse effects of the $\mathrm{I}^{249} \mathrm{M}^{280}$ variant on defense against RSV, we used $C \times 3 c r 1^{g f / / g f p}$ knockin mice (25), which lack functional CX3CR1 (referred to as $C X 3 C R 1^{-/-}$mice hereafter) (26), to determine whether loss of CX3CR1 recapitulates the severity of RSV-induced disease in newborn mice, as observed in humans with the $\mathrm{I}^{249} \mathrm{M}^{280}$ haplotype.

In this study, we show that CX3CR1 deficiency promotes lung pathology in RSV-infected newborn mice. We observed significantly more inflammation in both the airways and the lung parenchyma, accompanied by more mucus-producing cells and mucin gene expression in the lungs of $C X 3 C R 1^{-1-}$ mice, compared with that in WT mice. Examination of the immune cells infiltrating the lungs of the mice revealed 10- to 12 -fold more $\gamma \delta \mathrm{T}$ cells early p.i. and a greater frequency of IL- $17^{+} \gamma \delta \mathrm{T}$ cells in the $C X 3 C R 1^{-/-}$mice as compared with that in WT mice. The IL-17 response was a dominant overprotective IFN- $\gamma$ response early as well as later p.i. The lungs of CX3CR1-deficient mice expressed higher levels of IL-1 $\beta$ mRNA and protein, this cytokine being a potent inducer of IL-17 in $\gamma \delta$ T cells. Blockade of IL- $1 \beta$ signaling using IL-1 receptor antagonist significantly reduced IL- $17^{+} \gamma \delta \mathrm{T}$ cell numbers in the lungs of infected mice. In addition, blockade of IL-17RC abolished both the increased inflammatory response and mucus production in the lungs of $C X 3 C R 1^{-/-}$mice.

\section{Results}

$R S V$ infection of distal airways and alveolar epithelium in newborn mice. WT newborn mice were infected and examined for infection. As shown in Figure 1, RSV antigen ${ }^{+}$cells were sparse in the airways but were prominent in the alveoli of the newborn mice. This pattern of alveolar staining has been also detected upon histological evaluation of lung tissue recovered through autopsies of children who died from RSV-induced bronchiolitis (3).

CX3CR1 deficiency promotes lung pathology but does not affect viral burden after RSV infection. Given that the $\mathrm{I}^{249} \mathrm{M}^{280}$ mutation in CX3CR1 is associated with more hospitalizations of infants due to RSV-induced bronchiolitis, we compared the effect of RSV infection on lung pathology in newborn WT and $C X 3 C R 1^{-1}$ mice. 5-day-old pups were infected with RSV, and on day 7 after infection (p.i.), lungs were examined for mucus production and inflammation. The airways of $C X 3 C R 1^{-1-}$ mice displayed more inflammation and mucus-producing goblet cells as compared with those of WT mice (Figure 2, A and B). When analyzed for the expression of mucus-producing genes, significantly higher expression of Muc5ac and Gob5 mRNA was detected in the lungs of RSV-infected $C X 3 C R 1^{-/-}$pups compared with that in the WT pups (Figure 2C). However, mRNA expression of the L polymerase gene of RSV (Figure 2D) as well as the viral load (Figure 2E) in infected lungs was similar in WT and CX3CR1-deficient pups. We assessed some IFN-stimulated genes (ISGs) in the two groups of mice but did not detect any difference in expression (Supplemental Figure 1; supplemental material available online with this article; https://doi.org/10.1172/jci.insight.94605DS1). The expression of the ISGs Ifit 1 and Oasl2 in both WT and $C X 3 C R 1^{-1-}$ mice increased similarly in infected mice early p.i., and this increase was not evident later on day 7 p.i., which was expected, since ISGs are early response genes (Supplemental Figure 1). Neutrophil infiltration of the airways is a common feature in both infants infected by RSV (27) and in animal models of infection (28). We therefore examined neutrophil accumulation in the airways of the two groups of mice by immunohistochemistry. As shown in Figure 2, $\mathrm{F}$ and $\mathrm{G}$, neutrophils were readily detectable in the inflammatory infiltrate in the airways of infected $C X 3 \mathrm{CR}^{-1-}$ mice, and very few were detected in the airways of WT mice. Taken together, these data show that RSV infection induces more mucus production and airway inflammation in the absence of CX3CR1. 

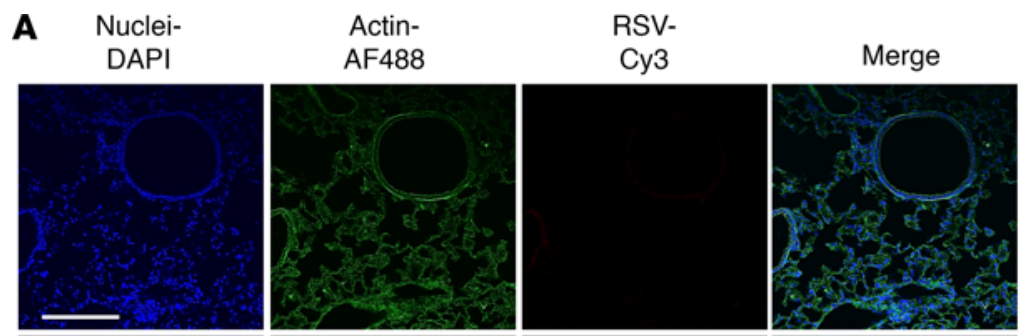

Naive
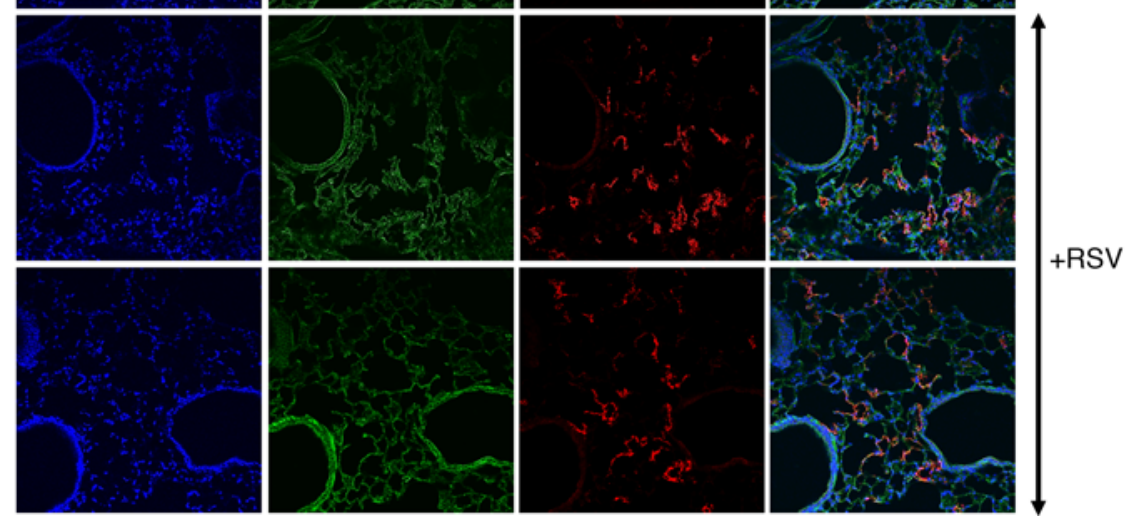

B

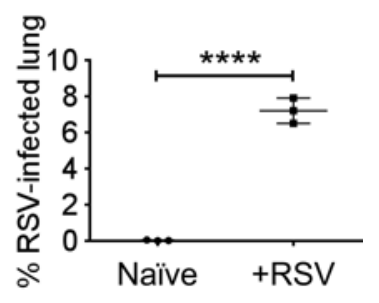

Figure 1. Intranasal infection with RSV results in viral localization to distal alveoli in mouse lungs. (A) 5 -day-old newborn mice were infected with $1 \times 10^{6} \mathrm{PFU}$ of RSV. On day 4 p.i., the mice were sacrificed and lungs were fixed, frozen, and processed for imaging. Immunofluorescence staining of frozen lung sections shows RSV localized to distal alveoli. Scale bar: $200 \mu \mathrm{m}$. (B) Quantitation was performed by assessing percentage of RSV-infected tissue in total tissue using Nikon Elements software. Data shown are mean \pm SEM, representative of 2 independent experiments; $n=3$ mice per group per experiment, ${ }^{* * * *} P<0.0001$. Student's unpaired $t$ test was used for statistical analysis.

CX3CR1 deficiency causes significantly greater accumulation of $I L-17^{+} \gamma \delta$ T cells in the lungs after RSV infection. We next examined the nature of the immune cells that infiltrate the airways of WT and CX3CR1-deficient newborn mice after RSV infection. Cell types and cytokines produced by the cells were examined by flow cytometry. The gating strategy is shown in Supplemental Figure 2. We performed these analyses at two time points after virus infection. One was on day 4 p.i., when we had previously noticed peak expression of the RSV large (RSV-L) polymerase gene in infected lungs of young mice (29). At this time point, the immune response to pathogen infection is dominated by cells of the innate immune system. We also performed analysis on day 7 p.i., which was suitable for studying immune response in both innate cells and $\mathrm{T}$ cells. On day 4 p.i., the total number of cells recovered from the lungs of both WT and $C X 3 C R 1^{-1-}$ mice was higher, but the numbers were not very different (Figure 3A). In our analyses, we focused on 3 innate immune cell types, NK cells, ILC2 cells, and $\gamma \delta \mathrm{T}$ cells, which are key cytokine-producing cells in the lung. The numbers of NK cells slightly increased p.i., but no statistically significant difference was noted among the groups (Figure 3A). Interestingly, we reproducibly observed a significantly higher number of $\gamma \delta \mathrm{T}$ cells (10- to 12 -fold more) in the lungs of infected $C X 3 C R 1^{-1-}$ mice compared with that in infected WT mice (Figure $3 \mathrm{~A}$ ). The frequency of IL- $17^{+} \gamma \delta \mathrm{T}$ cells was higher in infected WT and $C X 3 C R 1^{-I^{-}}$mice compared with that in uninfected mice, but it reached statistical significance only in the $C X 3 C R 1^{-1-}$ mice (Figure $3 \mathrm{~B}$ ). The frequency of IFN- $\gamma^{+} \gamma \delta \mathrm{T}$ cells was the reverse, being lower in infected $C X 3 C R 1^{-/-}$mice compared with WT mice (Figure 3B). In the case of ILC2 cells, we detected slightly lower numbers in uninfected $C X 3 \mathrm{CR}^{-1-}$ mice, but p.i. the numbers were similar in the two groups, showing an increase in ILC2 cell numbers in infected $C X 3 C R 1^{-1-}$ mice (Figure $3 \mathrm{~A}$ ). We assessed expression of IL-13 in ILC2 cells and of the cytokines IFN- $\gamma$ and IL-17 in both NK cells and $\gamma \delta \mathrm{T}$ cells by intracellular cytokine-staining techniques. The frequency of IL-13+ ILC2 cells in the lungs of WT mice increased upon infection but did not appreciably increase in the lungs of $C X 3 C R 1^{-1-}$ mice p.i., and the difference between the two groups of infected mice reached statistical significance (Supplemental Figure 3A). The frequency of IL-17 $7^{+} \mathrm{NK}$ cells was similar in the two groups, but that of IFN- $\gamma$-expressing NK cells was lower in the $C X 3 C R 1^{-/-}$mice (Supplemental Figure $3 \mathrm{~A}$ ). When we computed the absolute numbers, because of the $>10$-fold increase in the number of $\gamma \delta \mathrm{T}$ cells in the lungs of the $C X 3 C R 1^{-/-}$mice as well as an approximately 2-fold increase in the frequency of IL-17-expressing cells, the total number of IL- $17^{+} \gamma \delta \mathrm{T}$ cells in the lungs of infected $C X 3 C R 1^{-/-}$mice was 


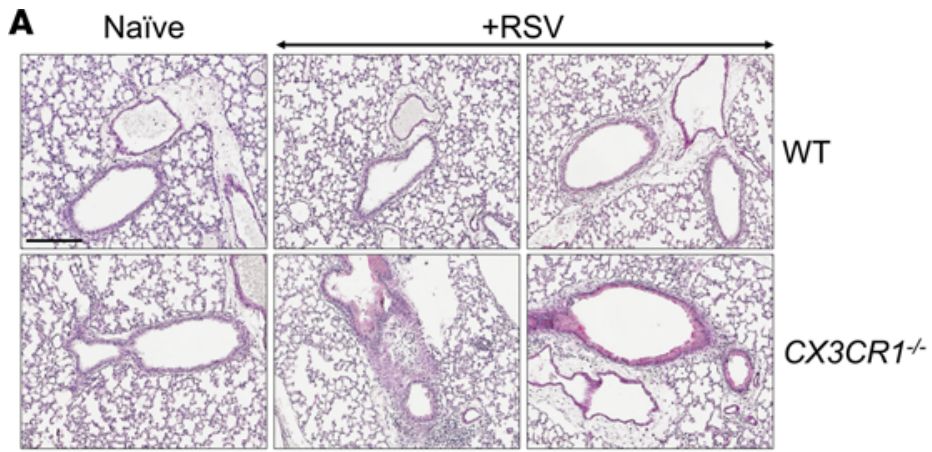

B
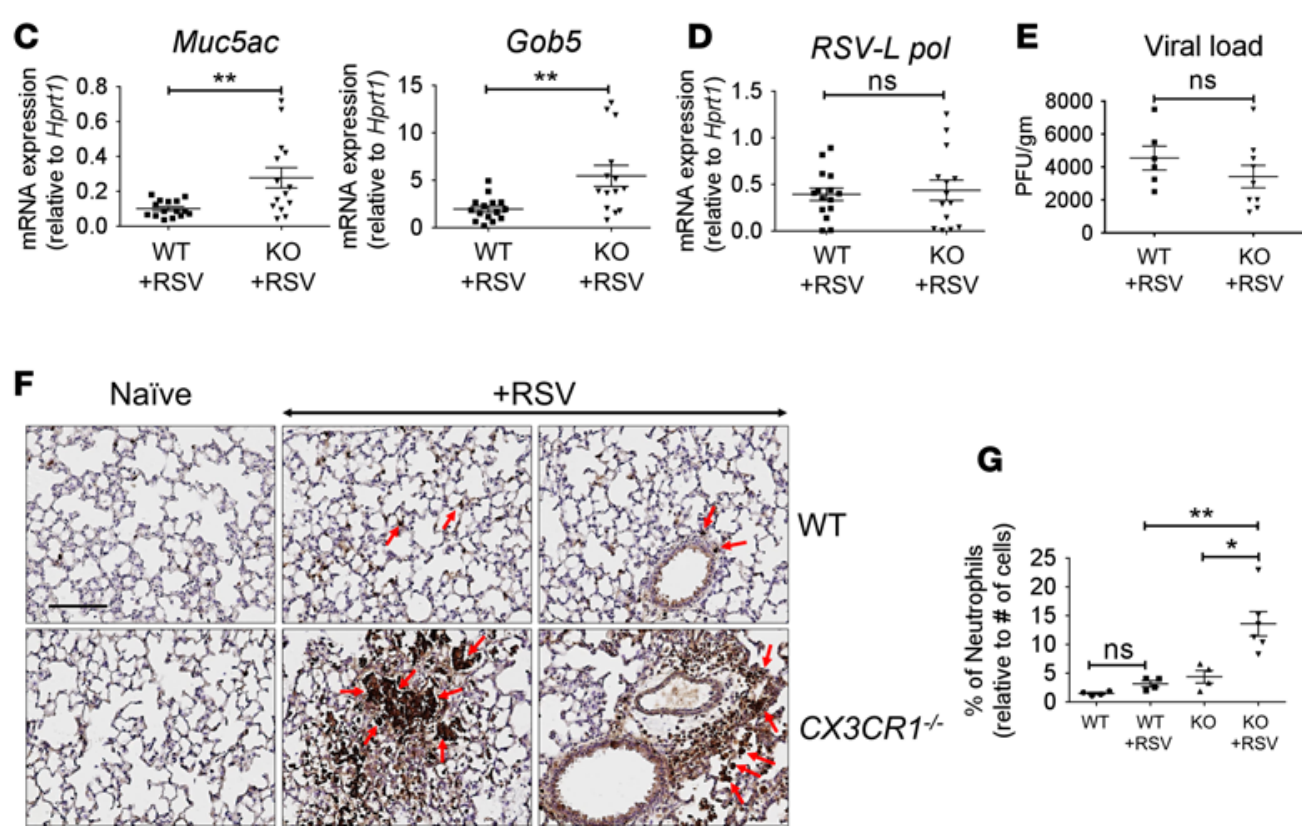

Figure 2. CX3CR1 deficiency promotes mucus production and neutrophil accumulation in the lungs but does not influence viral burden after RSV infection. (A) Histological assessment of lung sections after PAS staining showing mucus in the airways of WT and CX3CR $1^{-1-}$ newborn mice on day 7 p.i. by RSV. Original magnification, $\times 10$. Scale bar: $200 \mu \mathrm{m}$. (B) Pathological score showing mucus-producing goblet cells in the mentioned groups. CX3CR $1^{-1-}$ mice are referred to as KO. Data shown are mean \pm SEM combined from 2 independent experiments, $n=2-3$ mice per group per experiment. (C) mRNA expression of mucus-producing genes (Muc5ac and Gob5) and (D) RSV-L polymerase in lungs measured by quantitative RT-PCR on day 7 p.i. Data shown are mean \pm SEM of 2 independent combined experiments; $n=6-9$ mice per group per experiment. (E) Viral load in total lungs on day 7 p.i. detected by plaque assay using Vero cells. Data shown are mean \pm SEM combined from 2 independent experiments, $n=3-5$ mice per group per experiment. (F) Detection of neutrophils by immunohistochemical staining of lung sections on day 7 p.i. Neutrophils are indicated by red arrows. Original magnification, $\times 20$. Scale bar: $100 \mu \mathrm{m}$. (C) Quantitation of neutrophil accumulation normalized to the total number of cells present, as assessed using Nikon Elements software. Data shown are mean \pm SEM combined from 2 independent experiments; $n=2-3$ mice per group per experiment. ${ }^{*} P \leq 0.05,{ }^{* *} P \leq 0.01$. Kruskal-Wallis (B and $\mathbf{G}$ ) or Mann-Whitney $U$ test (C-E) was used for statistical analysis.

almost 20-fold higher (Figure 3C). In addition, because of the net increase in the number of $\gamma \delta \mathrm{T}$ cells in $C X 3 C R 1^{-/-}$mice, despite the lower frequency of IFN- $\gamma^{+} \gamma \delta \mathrm{T}$ cells, there was a 3- to 4-fold increase in the number of these cells in infected $C X 3 C R 1^{1^{--}}$mice compared with that in infected WT mice (Figure $3 C$ ). However, approximately 40 -fold more IL- $17^{+} \gamma \delta \mathrm{T}$ cells were present in the $C X 3 C R 1^{-/-}$mice compared with IFN- $\gamma^{+} \gamma \delta$ T cells (Figure 3C). Thus, the most remarkable consequence of CX3CR1 deficiency early p.i. was the accumulation of a high number of IL- $17^{+} \gamma \delta$ T cells in the lungs after RSV infection.

We next carried out a similar analysis on day 7 p.i., with the exception that we focused on $\gamma \delta \mathrm{T}$ cells and both $\mathrm{CD}^{+}$and $\mathrm{CD} 8^{+} \mathrm{T}$ cells at this time point. In the infected $C X 3 C R 1^{-1-}$ mice, although a surge in total $\gamma \delta \mathrm{T}$ cell numbers was not evident at this time (Figure 3D), as observed on day 4 p.i. (Figure $3 \mathrm{~A}$ ), we continued to observe a higher frequency as well as total number of IL-17+ $\gamma \delta$ T cells compared with that in WT mice, and the level of expression of IL-17 in these cells (as judged by geometric mean fluorescence intensity) was also higher (Figure 3, E and F). In contrast, in the case of IFN- $\gamma$, both the frequency and the 
A 4dpi
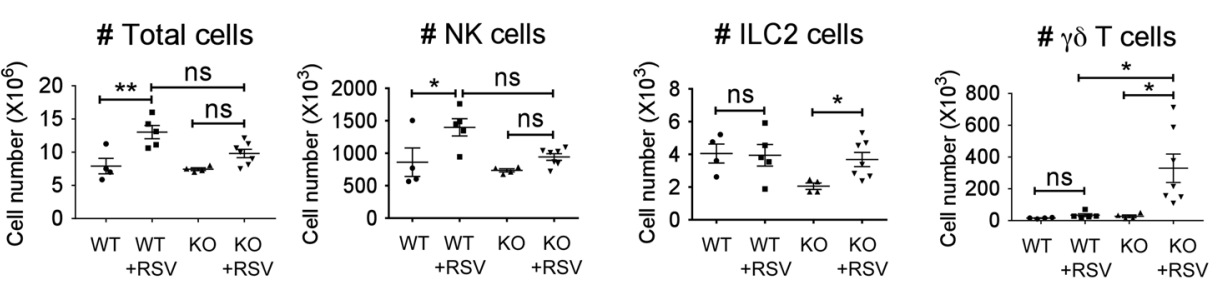

B $\%$ IL $-17+\gamma \delta$ T cells
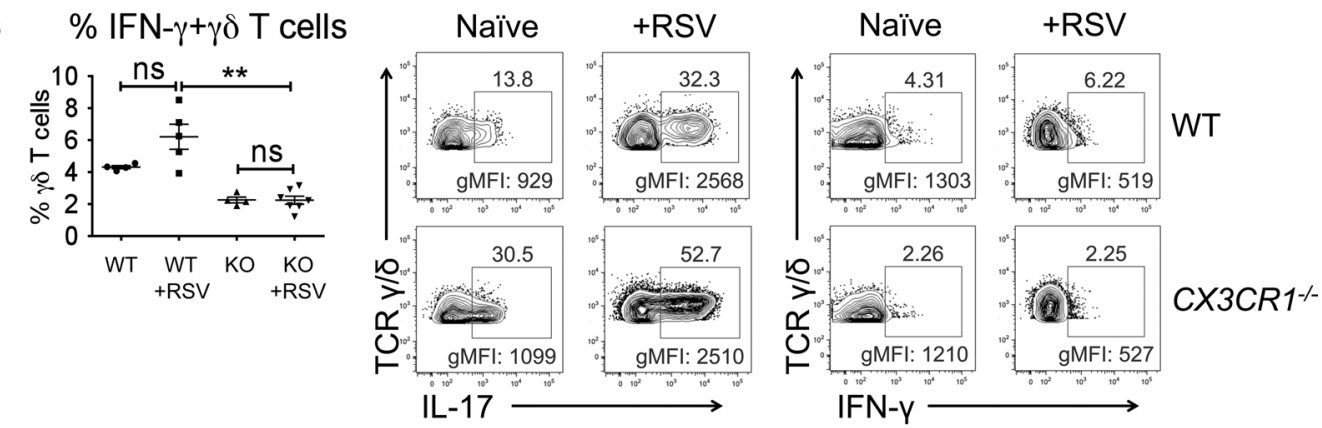

C
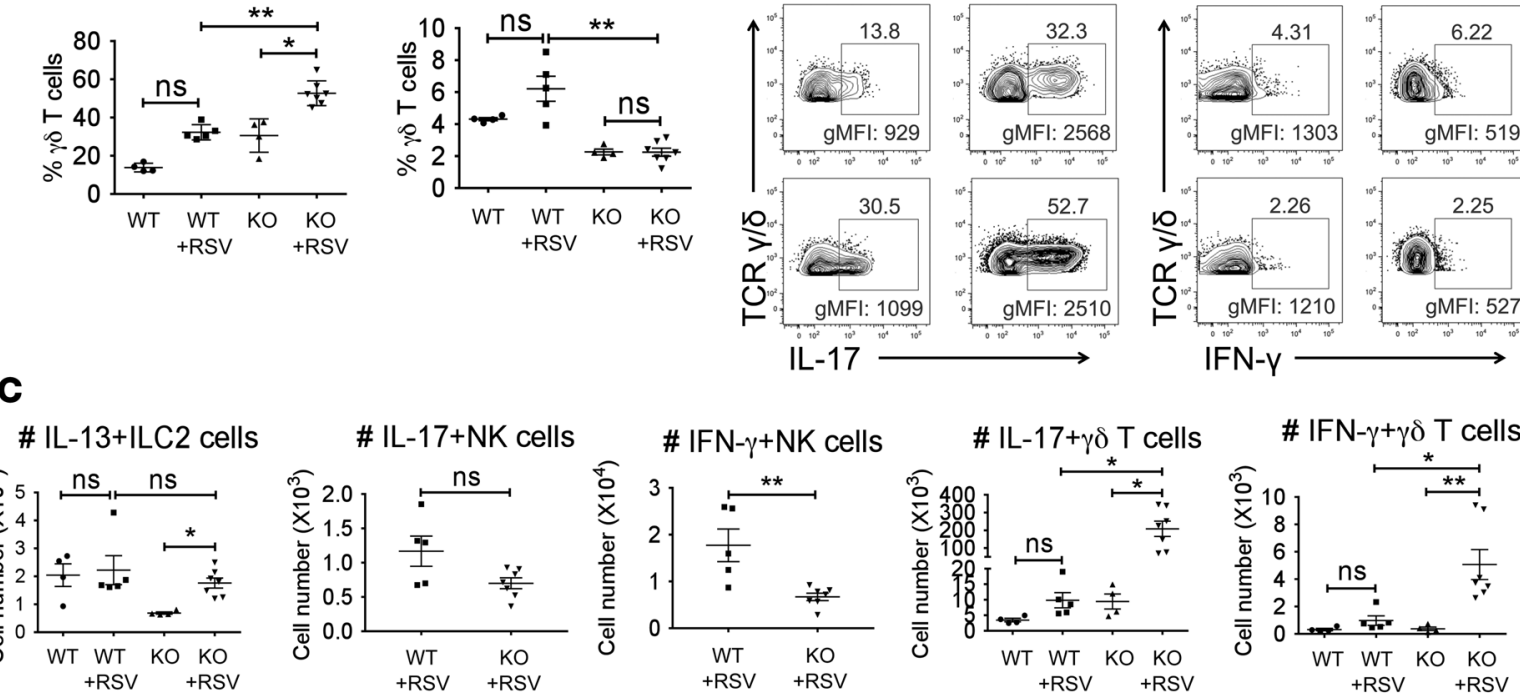

D

$7 \mathrm{dpi}$
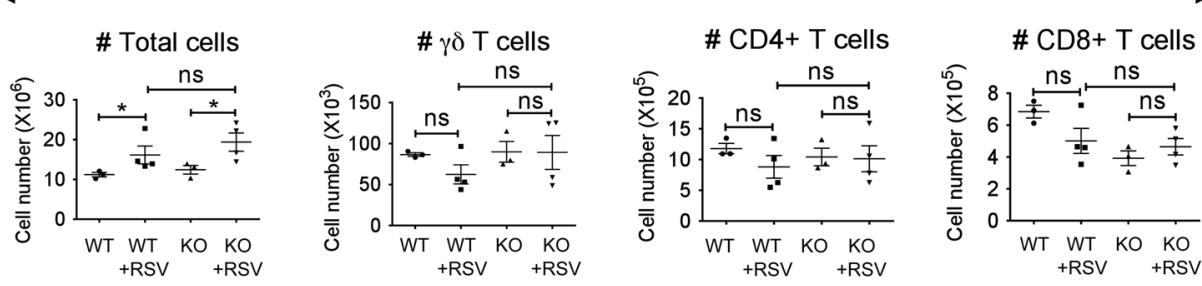

E $\% \mathrm{IL}-17+\gamma \delta \mathrm{T}$ cells
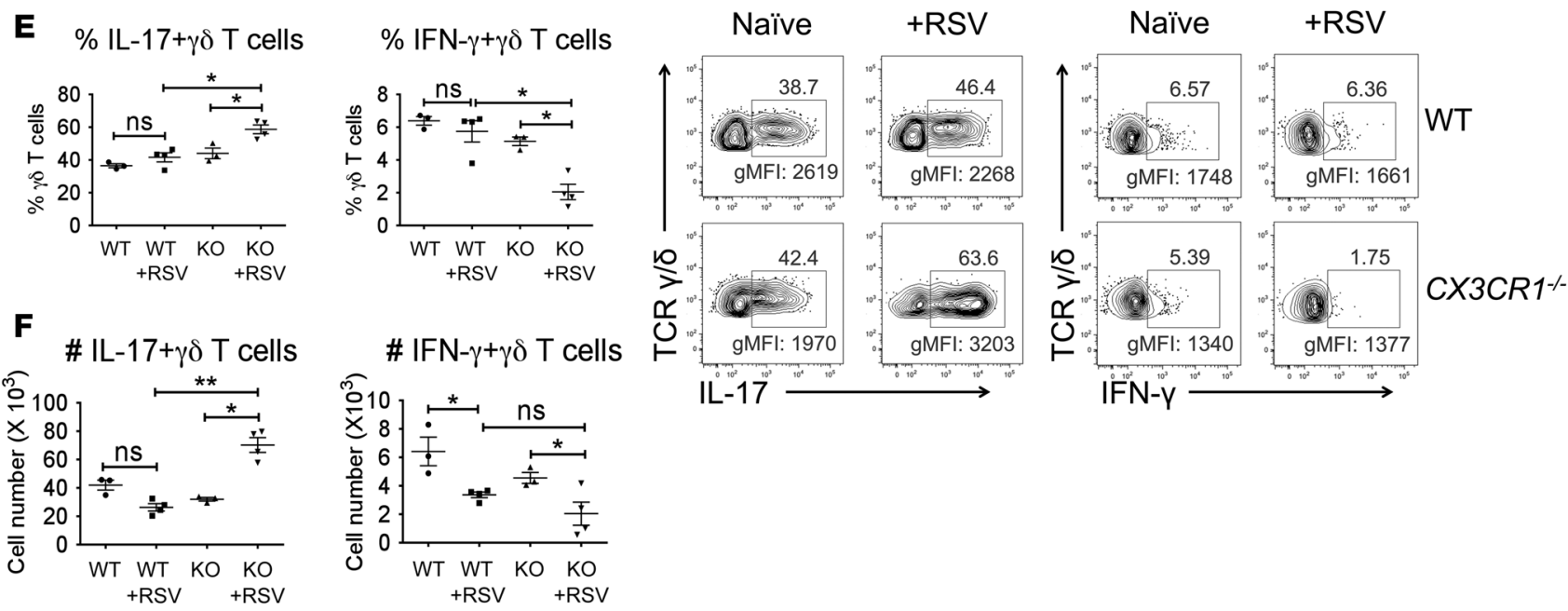

IFN-Y

Figure 3. CX3CR1 deficiency causes an increase in IL-17+ $\gamma \delta$ T cells and decrease in IFN- $\boldsymbol{\gamma}^{+} \boldsymbol{\gamma} \boldsymbol{\delta}$ T cells in lungs after RSV infection. Flow cytometric analysis of the mentioned groups showing (A) the total number of lung cells, NK cells, ILC2 cells and $\gamma \delta$ T cells; (B) percentages of IL-17+ and IFN- $\gamma^{+} \gamma \delta$ T cells, with representative contour plots; and (C) the total number of IL-13+ ILC2 cells, IL-17 ${ }^{+}$NK cells, IFN- $\gamma^{+}$NK cells, IL-17 $\gamma \delta$ T cells, and IFN- $\gamma^{+} \gamma \delta$ T cells on day 4 p.i. Data shown are mean \pm SEM, representative of 2 independent experiments; $n=4$ (uninfected) and 5-7 (RSV-infected) WT or KO mice per group 
per experiment. (D) Total number of lung cells, $\gamma \delta$ T cells, and CD4 $4^{+}$and CD8 $8^{+}$T cells. (E) Percentages of IL-17 $\gamma \delta$ T cells and IFN- $\gamma^{+} \gamma \delta$ T cells on day $7 p . i$. , with representative contour plots. (F) The total number of IL-17 $\gamma \delta$ T cells and IFN- $\gamma^{+} \gamma \delta$ T cells on day 7 p.i. Data shown are mean \pm SEM, representative of 2 independent experiments; $n=3$ (uninfected) and 4 (RSV-infected) WT or $C x 3 c r 1$ KO mice per group per experiment. Analyses were performed using Flowjo software. ${ }^{*} P \leq 0.05,{ }^{* *} P \leq 0.01$. Kruskal-Wallis (A-F) or Mann-Whitney U test (C) were used for statistical analysis.

absolute number of IFN- $\gamma^{+} \gamma \delta \mathrm{T}$ cells was lower in infected $C X 3 C R 1^{-/-}$mice compared with that in infected WT mice (Figure 3, E and F). Total numbers of $\mathrm{CD}^{+}$and $\mathrm{CD} 8^{+} \mathrm{T}$ cells were comparable in infected WT and $C X 3 C R 1^{-/-}$mice (Figure 3D). When cytokine expression in $\alpha \beta \mathrm{T}$ cells was examined, the frequencies and numbers of cytokine-expressing cells, particularly for IL-17 and IFN- $\gamma$, were lower in infected $C X 3 C R 1^{-/-}$mice (Supplemental Figure 3, B and C). In addition, there was statistically significant difference in IL-13-producing $\mathrm{CD}^{+}$or $\mathrm{CD} 8^{+} \mathrm{T}$ cells between the WT and $\mathrm{CX} 3 \mathrm{CR}^{-/-}$mice (Supplemental Figure 3, $\mathrm{B}$ and $\mathrm{C}$ ). Taken together, these data showed that, in the absence of functional CX3CR1, RSV infection results in more IL- $17^{+} \gamma \delta \mathrm{T}$ cells in the lungs of newborn mice but fewer IFN- $\gamma^{+}$cells whether among $\gamma \delta \mathrm{T}$ cells or $\mathrm{CD} 4^{+}$or $\mathrm{CD} 8^{+} \alpha \beta$ T cells.

Higher IL-1 $\beta$ expression in lungs of infected $C X 3 C R 1^{-1-}$ mice. We next investigated whether the lungs of $C X 3 C R 1^{-1-}$ mice express higher levels of Il1 $\beta$ mRNA compared with those of WT mice p.i., since this cytokine is a potent inducer of IL-17 in $\gamma \delta \mathrm{T}$ cells (30). As shown in Figure 4A, we detected a significantly higher level of Ill $\beta \mathrm{mRNA}$ in the lungs of infected $C X 3 C R 1^{-1-}$ pups as compared with that in the lungs of infected WT mice. At the protein level, we were able to detect IL-1 $\beta$ protein in most of the animals in the infected $\mathrm{CX}_{3} \mathrm{CR}^{-1^{--}}$group but not the infected WT group (Figure 4B). In addition, we also compared mRNA expression of $I l 6$ and Il23p19, which are known to be inducers of IL-17-producing $\gamma \delta \mathrm{T}$ cells. However, levels of $I l 6$ and Il23p19 mRNA remained unchanged on both days 4 and day 7 p.i. (Supplemental Figure 4, A and B). These data suggest that the absence of CX3CR1 renders pups of CX3CR1-deficient mice more prone to IL- $1 \beta$ production p.i., although the underlying mechanism is currently unclear.

Blocking IL-1 signaling reduces the frequency of $I L-17^{+} \gamma \delta T$ cells in virus-infected CX3CR $1^{-1-}$ mice. To understand the role of IL- $1 \beta$ in generating IL- $17^{+} \gamma \delta$ T cells, we next examined whether blocking IL-1 $\beta$ using an IL-1 receptor antagonist (IL-1RA; anakinra) has any effect on the percentage of $\mathrm{IL}-17^{+} \gamma \delta \mathrm{T}$ cells after RSV infection of $C X 3 C R 1^{-/-}$mice. To address this, we treated CX3CR1-deficent pups with IL-1RA, using 5 consecutive doses from day 5 to day 11, and infected them with RSV on day 5 four hours after administration of the first dose of IL-1RA (Figure 5A). On day 7 p.i., the total number of cells recovered from the lungs of infected $C X 3 C R 1^{-1-}$ mice treated with IL-1RA was significantly lower compared with that recovered from control mice that were only infected with RSV (Figure 5B). We detected a significant decrease in the IL- $17^{+} \gamma \delta \mathrm{T}$ cell population on day 7 p.i., both in frequency (Figure $5 \mathrm{C}$ ) and absolute numbers (Figure 5D) after IL-1RA treatment. In addition, the level of expression of IL-17 in these cells, as judged by geometric mean fluorescence intensity, was also lower after IL-1RA treatment (Figure 5C). However, no significant change was observed in the IFN- $\gamma^{+} \gamma \delta \mathrm{T}$ cells because of IL-1RA treatment (Figure 5, C and D). Taken together, these data suggest a role for IL-1 $\beta$ in promoting $\mathrm{IL}-17^{+} \gamma \delta \mathrm{T}$ cells in RSV-infected $C X 3 C R 1^{-/-}$mice.

Neutralization of IL-17RC causes decreased lung pathology after RSV infection. Given the increase in IL-17 ${ }^{+}$ $\gamma \delta \mathrm{T}$ cells in RSV-infected CX3CR1-deficent pups, we next investigated whether the increased mucus production and neutrophil infiltration in the lungs of mice were mediated by IL-17. To address this question, we treated $C X 3 \mathrm{CR}^{-/-}$pups with 2 doses of $\alpha \mathrm{IL}-17 \mathrm{RC}$-neutralizing antibody on days 2 and 5 after RSV infection (Figure 6A). We observed a marked decrease in mucus production and airway inflammation in the $C X 3 C R 1^{-1-}$ pups after blocking IL-17RC (Figure 6, B and C). In addition, neutrophil infiltration of the airways and the lung parenchyma was also reduced in the $\alpha \mathrm{IL}-17 \mathrm{RC}$-treated mice (Figure 6, D and E). Analysis of mucus-producing genes showed a significant decrease in the expression of Muc5ac and Gob5 mRNA in pups treated with $\alpha$ IL-17RC compared with that in pups treated with IgG control antibody (Figure 6F). However, neutralizing IL-17RC did not affect mRNA expression of RSV-L pol gene (Figure $6 \mathrm{G}$ ) or the viral load (Figure $6 \mathrm{H}$ ) in the infected pups. Taken together, the results of our study show that the absence of functional CX3CR1 causes an exaggerated IL-17 response in $\gamma \delta \mathrm{T}$ cells in newborn mice, which causes an increase in pulmonary immune pathology. In addition, our data suggest that the augmented mucus production and airway inflammation are not caused by the type 2 cytokine IL-13, produced by either ILC2 cells or T cells. 


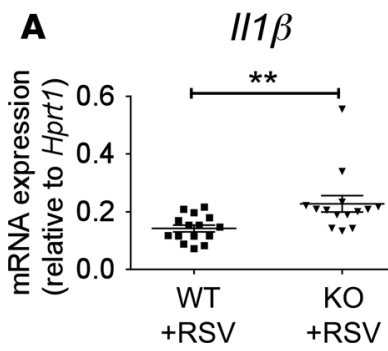

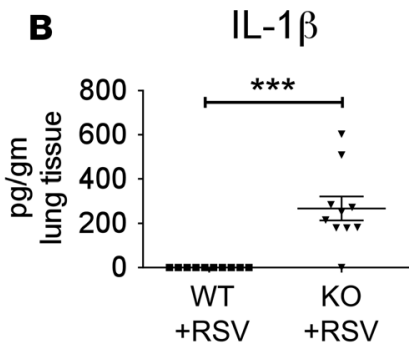

Figure 4. Increase in IL-1 $\beta$ level in the lungs of RSV-infected mice in the absence of CX3CR1. (A) mRNA and (B) protein expression corresponding to IL-1 $\beta$ in the lungs of WT and $C \times 3 C r 1 \mathrm{KO}$ mice assayed by quantitative RT-PCR and ELISA, respectively, on day 7 p.i. Data shown are mean \pm SEM combined from 2 independent experiments; $n=6-9$ mice per group per experiment (for mRNA) and 4-6 mice per group per experiment (for protein). ${ }^{* *} P \leq 0.01,{ }^{* *} P \leq 0.001$. Mann-Whitney $U$ test was used for statistical analysis.

\section{Discussion}

Our study of RSV infection of newborn CX3CR1-deficient mice recapitulated many features of RSV infection of infants. We detected infection of the alveolar epithelium, which was also observed upon histopathological evaluation of autopsies of infants who had died from RSV-induced severe bronchiolitis (3). The findings in this study highlight an important protective role of the chemokine receptor CX3CR1 in the context of RSV infection in infants. A previous study showed that the $\mathrm{I}^{249} \mathrm{M}^{280}$ mutation in CX3CR1, which is unable to bind its endogenous ligand, CX3CL1, is associated with more severe bronchiolitis in infected children (23). The calculated odds ratio suggested that children who carried the homozygous $\mathrm{I}^{249} \mathrm{M}^{280}$ mutation were twice as likely to be hospitalized for severe RSV-induced bronchiolitis as those carrying WT alleles (23). In the present study, we also show an increased propensity for lung pathology in infected newborn mice in the absence of functional CX3CR1. These mice show two important features of lung pathology observed in infants with RSV-mediated severe bronchiolitis-increased mucus production and pulmonary neutrophil infiltration. The altered immune response associated with absence of a functional CX3CR1 was found to be an increase in IL-17 $\gamma \delta$ T cells and a decrease in IFN- $\gamma^{+} \gamma \delta \mathrm{T}$ cells in the lungs of the newborn mice. Blockade of IL-17RC attenuated the increased lung pathology observed in $C X 3 C R 1^{-1-}$ mice.

RSV infection of mice has been studied using both BALB/c and C57BL/ 6 strains of mice. In addition, both RSV A2 and line 19 strains of RSV have been used in independent studies; both strains belong to the antigenic group A of RSV. Line 19, but not RSV A2, was previously shown to induce more mucus in adult $\mathrm{BALB} / \mathrm{c}$ mice in an IL-13-dependent fashion (31). In adult BALB/c mice infected by different clinical isolates of RSV (32) or by a chimeric RSV A2-line 19 strain of RSV (33), IL-13 and mucus production were associated with the F protein of RSV. These studies show that IL-13 mediates mucus production in response to line 19 in adult BALB/c mice. However, IL-17 was shown to be important for mucus production in adult C57BL/6 mice infected by line 19 (34). Our study of newborn $C X 3 C R 1^{-1-}$ C57BL/6 mice infected by RSV line 19 also shows dependence on IL-17 for enhanced mucus production and neutrophil accumulation in the lungs of infected mice. A previous study of infection of WT and CX3CR1-deficient adult C57BL/6 mice showed slightly fewer neutrophil numbers in the bronchoalveolar lavage fluid of CX3CR1-deficient mice compared with WT mice when examined on day 4 p.i. (35). However, in a different study by the same group using RSV A2 and BALB/c mice, instillation of a neutralizing monoclonal antibody against the G protein of RSV on day 5 p.i. actually doubled pulmonary neutrophil accumulation when the mice were examined 2 days later on day 7 (14). This observation resembles our finding of increased neutrophil accumulation in the lungs of RSV line 19-infected newborn $C X 3 C R 1^{-/-}$mice on day 7 p.i. Collectively, these studies suggest that blocking the $\mathrm{G}$ protein-CX3CR1 interaction using anti-G protein antibody or deletion of the $C \times 3 c r 1$ gene has a deleterious effect on day 7 after viral infection in both adult and newborn mice, irrespective of the genetic background of mice. In newborn mice, absence of CX3CR1 promotes both IL-17 expression and mucus production also at an earlier time p.i. on day 4 as also observed on day 7 p.i. This may be due to the dominant contribution of $\gamma \delta$ T cells to IL-17 expression in newborn mice, as demonstrated in our study. It is well recognized that $\gamma \delta$ T cells play an important role in neonatal protection against pathogens with the ability to mount a rapid response to infectious agents $(36,37)$.

The question arises of why absence of functional CX3CR1 triggers an increase in both the frequency of IL- $17^{+} \gamma \delta \mathrm{T}$ cells in RSV-infected newborn mice and their numbers early p.i. We observed that absence of functional CX3CR1 caused an increase in the level of the inflammatory cytokine IL-1 $\beta$ in the lungs of the infected mice. IL-1 $\beta$ is a well-recognized inducer of IL-17 in $\gamma \delta$ T cells (30), and we observed higher frequency and numbers of IL- $17^{+} \gamma \delta \mathrm{T}$ cells on both days 4 and 7 after RSV infection of the $C X 3 C R 1^{-/-}$mice. A previous study suggested a role for IL-1 $\beta$ in increasing the pool size of IL-1 $7^{+} \gamma \delta$ T cells in LPS-treated 
A

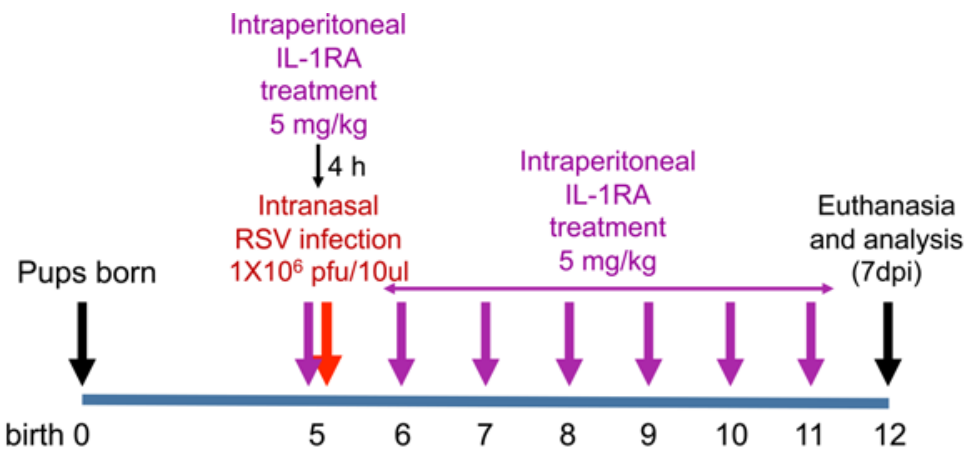

Day after birth 0

5
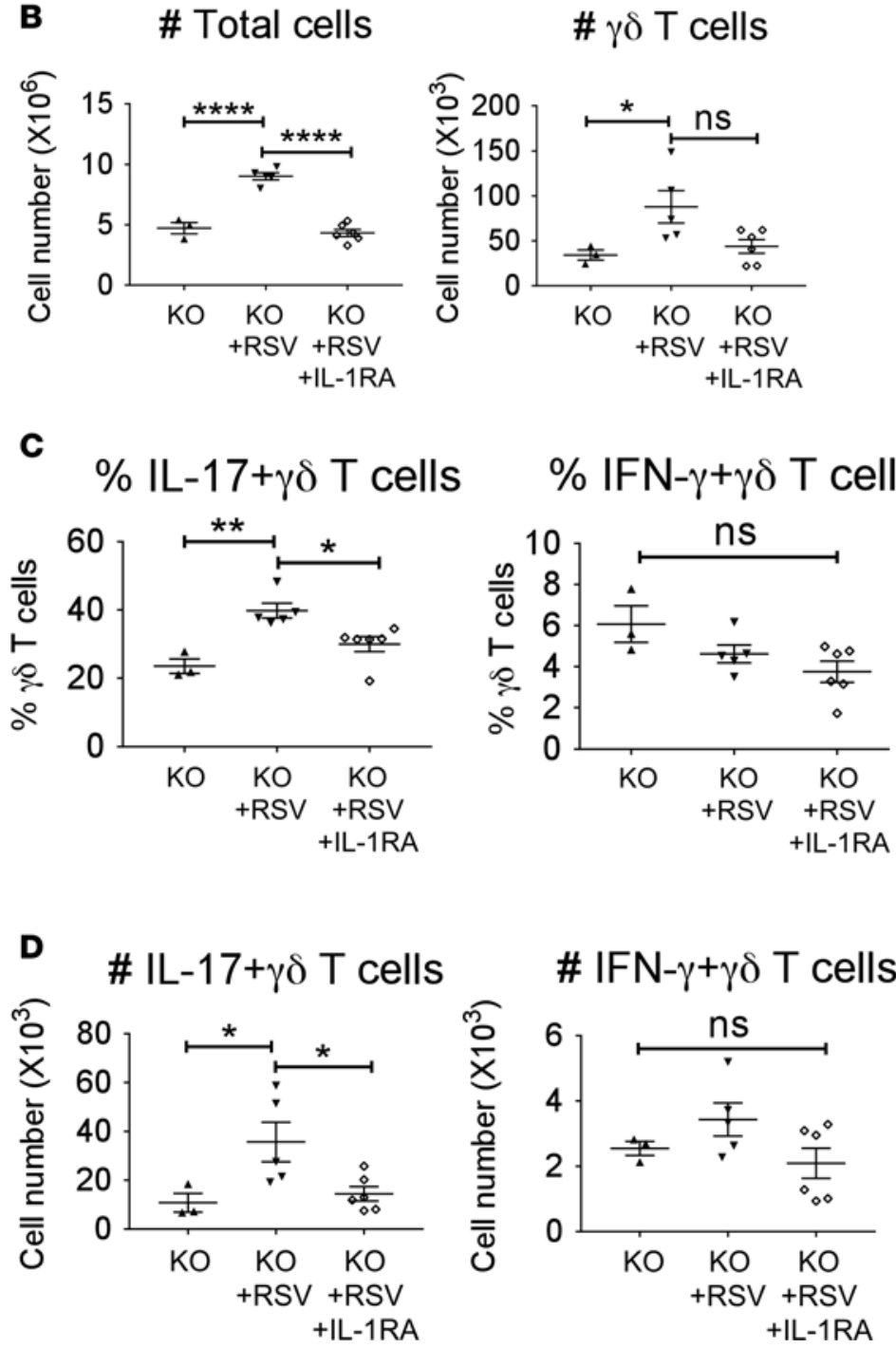

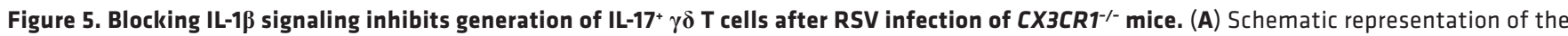
experimental plan. Neonatal mice were treated with IL-1RA (anakinra) at a dose of $5 \mathrm{mg} / \mathrm{kg}$ by the intraperitoneal route every 24 hours as shown. The pups were infected with $1 \times 10^{6} \mathrm{PFU}$ of RSV by the intranasal route 4 hours after the first IL-1RA treatment on day 5 , and mice were sacrificed on day 7 p.i. for analysis. (B-D) Flow cytometric analysis of the mentioned groups, showing (B) the total number of lung cells and $\gamma \delta \mathrm{T}$ cells; (C) percentages of IL-17+ and IFN- $\gamma^{+} \gamma \delta$ T cells, with representative contour plots, and (D) the total number of IL-17 $\gamma \delta$ T cells and IFN- $\gamma^{+} \gamma \delta$ T cells on day 7 p.i. Analyses were performed using Flowjo software. Data shown are mean $\pm S E M$, representative of 2 independent experiments; $n=3$ (uninfected), 5 (RSV-infected), and 6 (RSV-infected + IL-1RA) mice per group per experiment. ${ }^{*} P \leq 0.05,{ }^{* *} P \leq 0.01$, ${ }^{* * *} P \leq 0.0001$. 1-way ANOVA with Tukey's post-hoc test (B-D) was used for statistical analysis. 
A

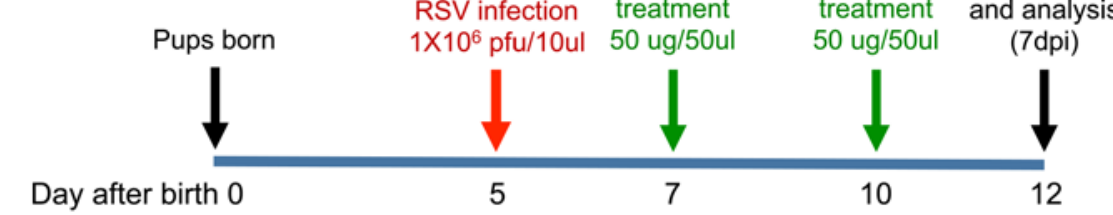

B

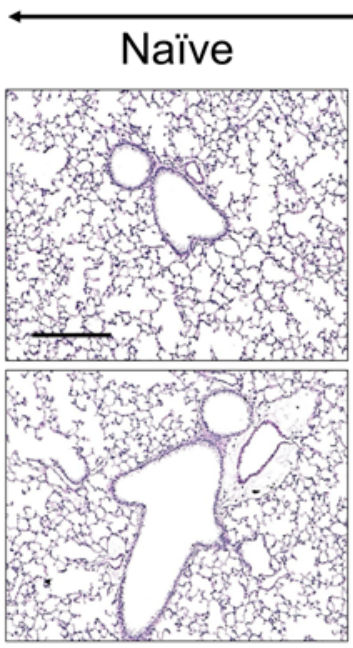

CX3CR $1 \%$

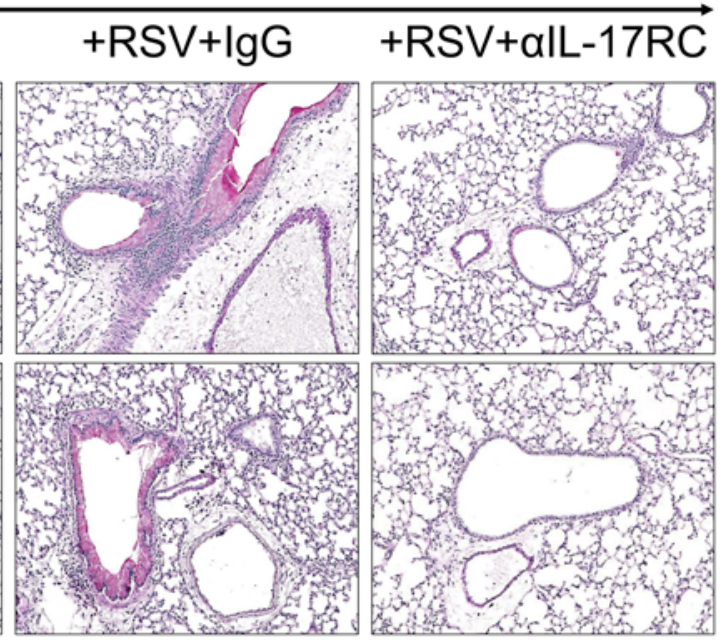

C

\section{Mucus-producing goblet cells}

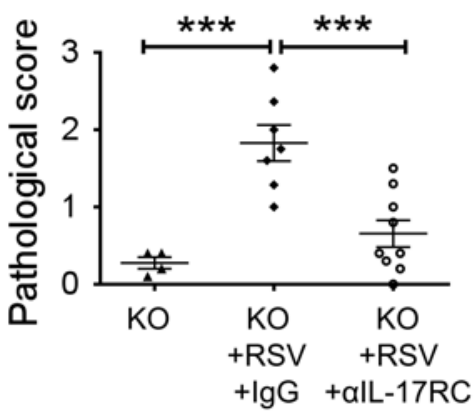

D

CX3CR $1 \%$
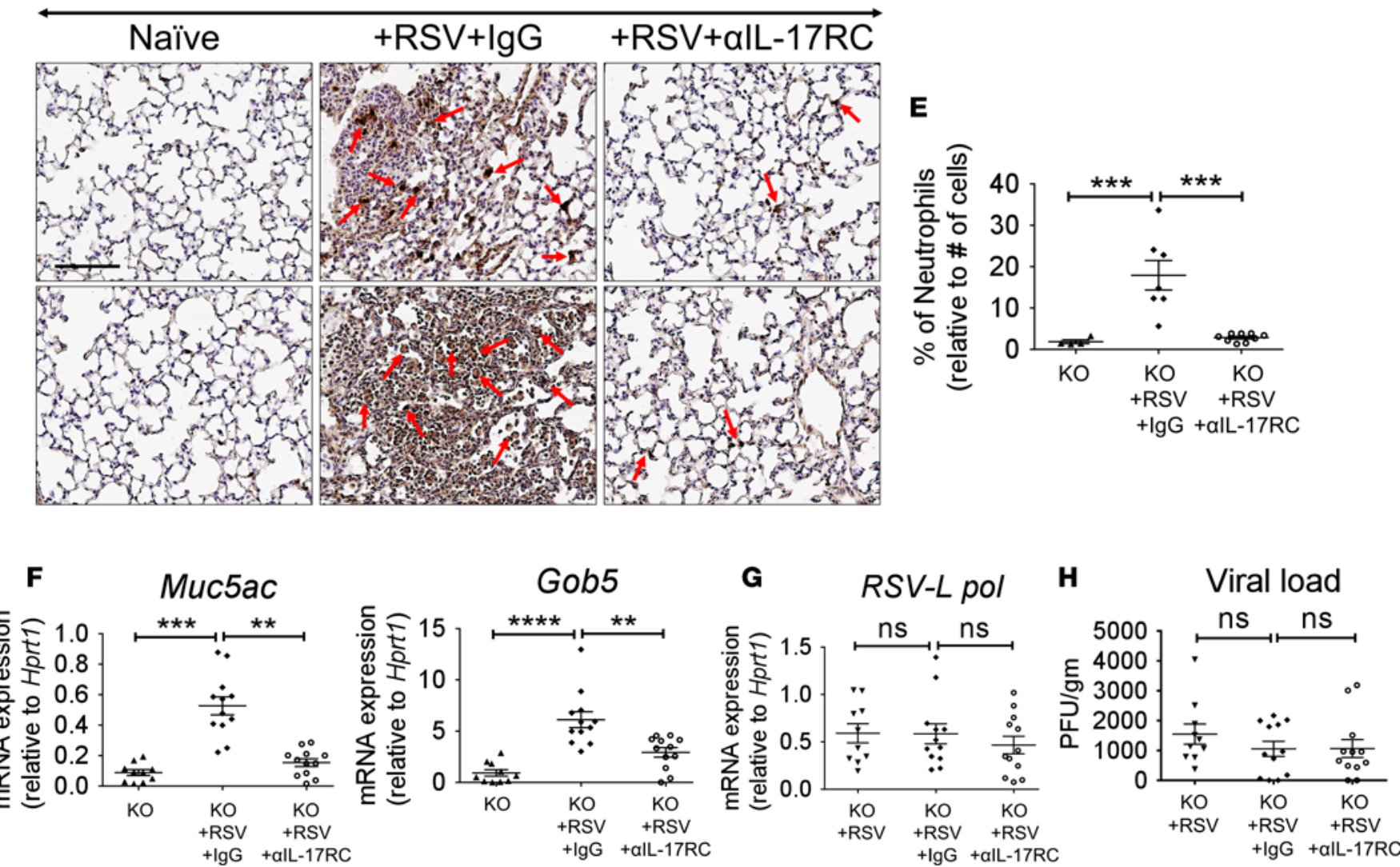

Figure 6. Neutralization with $\alpha$ IL-17RC reduces lung pathology in RSV-infected CX3CR1-/- mice. (A) Schematic representation of the experimental plan. 5-day-old pups were infected with $1 \times 10^{6} \mathrm{PFU}$ of RSV and treated with two doses of $50 \mu \mathrm{g}$ alL-17RC on days 2 and 5 p.i., and mice were sacrificed on day 7 p.i. (B) Histological assessment of lung sections after PAS staining to detect mucus in the airways of the $C X 3 C R 1^{-1-}$ pups treated with either control lgG or 
$\alpha$ IL-17RC on day 7 p.i. Original magnification, $\times 10$. Scale bar: $200 \mu \mathrm{m}$. (C) Pathological score showing mucus-producing goblet cells in the mentioned groups. Data shown are mean \pm SEM combined from 2 independent experiments; $n=2-5$ mice per group per experiment. (D) Detection of neutrophils by immunohistochemical staining of lung sections on day 7 p.i. Neutrophils are indicated by red arrows. Original magnification, $\times 20$. Scale bar: $100 \mu \mathrm{m}$. (E) Quantitation of neutrophil accumulation normalized to the total number of cells present, as assessed using Nikon Elements software. Data shown are mean $\pm \mathrm{SEM}$ combined from 2 independent experiments; $n=2-5$ mice per group per experiment. (F) mRNA expression of mucus-producing genes (Muc5ac and Gob5) and (G) RSV-L polymerase in the lungs measured by quantitative RT-PCR on day 7 p.i. (H) Viral load in total lungs on day 7 p.i. detected by plaque assay using Vero cells. Data shown are mean \pm SEM combined from 2 independent experiments; $n=5-6$ mice per group per experiment. ${ }^{* *} P \leq 0.01,{ }^{* * *} P \leq 0.001$, ${ }^{* * * *} P \leq 0.0001$. 1-way ANOVA with Tukey's post-hoc test (C, $\mathbf{E}$, and $\left.\mathbf{F}-\mathbf{H}\right)$ was used for statistical analysis.

mice (38). IL-1 $\beta$ can induce the expression of the chemokine CCL20 in AECs and in fetal lungs (39). The chemokine CCL20 was shown to promote accumulation of $\mathrm{IL}-17^{+} \gamma \delta \mathrm{T}$ cells via its cognate receptor CCR6 during liver injury (40). In RSV-infected mice, neutralization of CCL20 reduced virus-induced lung pathology by blocking recruitment of dendritic cells to the lungs of the mice (41). Thus, the IL-1 $\beta$-mediated increase in CCL20 expression early p.i. may be responsible for driving the accumulation of $\gamma \delta \mathrm{T}$ cells in $\mathrm{CX}_{3} \mathrm{CR}^{1^{-1-}}$ mice, which would otherwise be suppressed by the CX3CL1/CX3CR1 axis. Taken together, the reduction in the numbers of both total $\gamma \delta \mathrm{T}$ cells and IL-17+ $\gamma \delta \mathrm{T}$ cells upon IL-1RA treatment in our study suggests a primary role for IL- $1 \beta$ in both expansion of the $\gamma \delta \mathrm{T}$ cells as well as in IL-17 expression by the cells. As observed in our study, increased IL-1 $\beta$ and IL-17 secretion in the lungs of the $C X 3 C R 1^{-/-}$mice would be expected to promote neutrophil recruitment to the lungs, given that collaboration between IL-17 and cytokines, such as IL-1 $\beta$ and TNF- $\alpha$, induces expression of neutrophil-attracting chemokines $(30,42)$.

The primary source of type I IFN after RSV infection has been shown to be alveolar macrophages, which help to recruit inflammatory monocytes to the lungs to mount an antiviral defense, although alveolar macrophages are unable to support replication of RSV (43). It is possible that alveolar macrophages and infiltrating monocytes in the absence of functional CX3CR1 are more prone to producing IL-1 $\beta$ in virus-infected lungs. Production of IL-1 $\beta$ requires two signals. One signal stimulates pro-IL-1 $\beta$ transcription (via TLR signaling), while the second (best studied via ATP-P2X7 receptor interaction) promotes IL-1 $\beta$ secretion via caspase-1-mediated cleavage of pro-IL-1 $\beta$ (44). In a model of macular degeneration, infiltrating mononuclear cells from CX3CR1-deficient mice were found to upregulate P2X7 expression with an increase in IL- $1 \beta$ secretion causing retinal damage (45). These data collectively suggest that CX3CR1 expression in monocytes and macrophages helps suppress IL-1 $\beta$ production to limit IL-17 production from lung $\gamma \delta \mathrm{T}$ cells in the context of RSV infection. The precise mechanism downstream of CX3CR1 that regulates IL-1 $\beta$ production in the lungs of RSV-infected newborn mice remains to be determined.

Our findings suggest a pathogenic role of IL-17-producing $\gamma \delta \mathrm{T}$ cells in the context of CX3CR1 deficiency and RSV infection. Similarly, in a model of RSV F protein-mediated vaccination and challenge with RSV, $\gamma \delta \mathrm{T}$ cells were found to be pathogenic, since their depletion attenuated disease severity (46). In an earlier study (47), $\gamma \delta \mathrm{T}$ cells from neonatal RSV-infected BALB/c mice were found to be deficient in IL-17 production compared with cells isolated from adult mice. In addition, IL-17 administration in neonates reduced inflammation, including neutrophil numbers, in the bronchoalveolar lavage fluid without any effect on mucus production. Since IL-17 normally promotes neutrophil recruitment, the reduction of neutrophils with IL-17 in this study may have been specific to the model or because only IL-17 was administered without IL-1 $\beta$. What is common between this study and ours is that we also did not observe an appreciable increase in IL-17 or IL-1 $\beta$ above baseline in WT neonates, our mice being on C57BL/ 6 background. The enhanced IL-17 response in $\gamma \delta \mathrm{T}$ cells and increase in IL-1 $\beta$ levels in the lungs promoting a strong neutrophilic inflammatory response and mucus production were only observed in the absence of functional CX3CR1, which resembled that observed in RSV-infected children harboring the $\mathrm{I}^{249} \mathrm{M}^{280}$ mutation in CX3CR1 (23). Given that neutralization of IL-17 in the $C X 3 C R 1^{-1-}$ pups prevented the neutrophilic inflammation and mucus production, it appears that CX3CR1 insufficiency renders both newborn mice and infants susceptible to a more severe inflammatory state in which IL-17 plays a central pathogenic role. In addition to the IL-17-promoting effect of CX3CR1 deficiency, we also observed an IFN- $\gamma$-dampening effect in the $C X 3 C R 1^{-/-}$mice in both $\gamma \delta \mathrm{T}$ cells and $\alpha \beta \mathrm{T}$ cells on day $7 \mathrm{p} . \mathrm{i}$. IFN- $\gamma$ is considered to be a protective cytokine in the context of RSV infection. Pretreatment with CpG oligodeoxynucleotide in newborn mice induces a protective Th1 (IFN- $\gamma$ ) response with reduced disease upon subsequent RSV infection (48). Thus, the combined effect of enhanced IL-17 response and reduced IFN- $\gamma$ production from immune cells would be expected to promote disease severity in the absence of functional CX3CR1, as observed in our analyses. 
An earlier study reported enhanced Th2 polarization in the context of tlr4 mutation as a cause of RSV-mediated bronchiolitis (49). A recent report has associated increased production of the immunosuppressive cytokine IL-10 from regulatory B cells (Bregs) with increase in RSV-induced disease severity in $50 \%$ of the study cohort (50). In this study, the authors observed a positive correlation between frequencies of Bregs in the blood and viral load in nasal swabs of the infants (50). When considered in aggregate, the finding of RSV-induced severe bronchiolitis in infants carrying a mutant CX3CR1 receptor (23) and our present study of RSV infection of $C X 3 C R 1^{-/-}$mice, aberrant IL-17 production appears to be the disease-driving cytokine in the absence of a functional CX3CR1 receptor. However, we did not observe any significant effect of CX3CR1 deficiency on viral load. As reviewed recently, disease severity caused by RSV infection may be dependent or independent of viral load (6). Collectively, these observations suggest that severe bronchiolitis induced by RSV infection can be caused by different immune responses, which may be influenced by the genetic makeup of the host.

Our study has identified a pathogenic role for $\gamma \delta \mathrm{T}$ cells in the context of specific immune deficiencies, such as lack of CX3CR1 in early life, during which these cells normally are considered to have a host-protective function when adaptive immunity has not yet reached full maturity, particularly with respect to IFN- $\gamma$ response from $\alpha \beta$ T cells. Since RSV infection is a common feature in almost all children, some of whom develop severe bronchiolitis requiring hospitalization, those who harbor mutations in CX3CR1 may benefit from treatment modalities targeting the IL-17R. RSV infection resulting in severe bronchiolitis also increases the risk for developing respiratory disorders, such as recurrent wheezing and asthma (6). We propose that, in infants harboring the $\mathrm{I}^{249} \mathrm{M}^{280}$ mutation in CX3CR1, targeting the IL-17R may achieve the dual benefit of both stemming severity of illness in early life and reducing the risk for asthma in later life.

\section{Methods}

Mice. B6.129P-Cx3cr1tm1Litt/J mice (catalog 005582), which are homozygous for the CX3CR1-GFP targeted mutation (referred to here as $C X 3 C R 1^{-1-}$ mice) and its control C57BL/6J mice (catalog 000664) were purchased from The Jackson Laboratory. Mice were housed and bred under pathogen-free conditions in the Department of Laboratory Animal Resources at the University of Pittsburgh to generate neonatal mice. 5-day-old neonatal mice were used for all experiments irrespective of their sex. No sex bias in immune response was observed in our experiments.

Virus culture and titration. The RSV line 19 was cultured and amplified in the HEp-2 cell line (ATCC CCL-23), as described previously (51). Briefly, HEp-2 cells were infected with the virus and then maintained in culture for 3 days. Cells were lysed by sonication, and the cell supernatant containing released virus was collected for experiments. The virus titration was then done by plaque assay using NY3.2 STAT1 ${ }^{-/-}$fibroblast cells (gift of Joan E. Durbin, New York University School of Medicine, New York, New York, USA) in 24-well tissue culture plates precoated with polyethylenimine (Sigma-Aldrich, catalog 408727). Before infection, the culture medium was removed from the wells, virus stock was serially diluted, and each dilution was added to duplicate wells. The virus stock in EMEM medium was allowed to adsorb to the cell monolayer for 2 hours with constant shaking at $37^{\circ} \mathrm{C}$. Adsorption of virus was stopped by adding $1 \%$ methylcellulose (Sigma-Aldrich, catalog 56340) overlay medium, followed by incubation at $37^{\circ} \mathrm{C}$ and $5 \% \mathrm{CO}_{2}$ for 3 days. Viral plaques were then developed for counting using goat anti-RSV all antigens primary antibody (Meridian Life Science, catalog B65860G), donkey anti-goat IgG AP secondary antibody (Santa Cruz Biotechnology, catalog sc-2022), and BCIP/NBT alkaline phosphatase substrate (Sigma-Aldrich, catalog B5655).

$R S V$ infection and treatment of newborn mice. 5-day-old neonatal mice were infected with $1 \times 10^{6} \mathrm{PFU}$ of RSV line 19 (gift of Martin L. Moore, Emory University, Atlanta, Georgia, USA) in $10 \mu 1$ of EMEM culture medium by the intranasal route and then sacrificed on day 4 or 7 p.i. Infected pups were treated with $50 \mu$ g goat anti-mIL-17RC antibody (R\&D Systems, catalog AF2270) or goat IgG isotype control (R\&D Systems, catalog AB-108-C) by the intraperitoneal route on days 2 and 5 p.i. To block IL-1 $\beta$ effects, neonatal mice were treated with IL-1RA (anakinra, Kineret) (Amgen, NDC 66658023407, purchased from University of Pittsburgh Medical Center pharmacy) at a dose of $5 \mathrm{mg} / \mathrm{kg}$ by the intraperitoneal route every 24 hours starting from day 5 up to day 11 . The pups were infected with $1 \times 10^{6} \mathrm{PFU}$ of RSV line 19 by the intranasal route 4 hours after the first IL-1RA treatment on day 5.

The right multilobe lung was fixed in SafeFix II (Fisher Healthcare, catalog 23-042-601) and processed for histological studies. The left single lobe was homogenized in OptiMEM medium (Gibco, catalog 31985070), and the homogenate was used to detect either viral load or cytokine mRNA or protein levels. 
Lung viral load estimation. Viral load in the lungs was estimated by plaque assay using Vero cells (ATCC CCL-81) as previously described (52). Briefly, lung supernatant was collected after centrifugation of the lung homogenate and immediately processed for plaque assay. 2- and 10-fold dilutions of lung supernatants were made in OptiMEM and applied to monolayers of Vero cells in 24-well tissue culture plates. After 1 hour of incubation at room temperature with constant shaking, the inoculum was replaced with methylcellulose-supplemented L-15 medium (2\% methylcellulose, Sigma-Aldrich, catalog 56340) mixed 1:1 with $2 \times$ L-15 medium (Lonza, catalog 12-669E) supplemented with 2\% FBS, 4 mM L-glutamine (Gibco, catalog 25030-081), and $200 \mathrm{U}$ penicillin with $200 \mu \mathrm{g} / \mathrm{ml}$ streptomycin (Gibco, catalog 15140-122). Cells were incubated at $35^{\circ} \mathrm{C}$ and $5 \% \mathrm{CO}_{2}$ for 7 days. Overlay medium was then aspirated, and cells were immunostained with goat antiRSV antibody (EMD Millipore, catalog AB112), followed by HRP-conjugated donkey anti-goat IgG (Jackson Immunoresearch, catalog 705-035-147). Red-colored plaques were developed with 3-amino-9-ethylcarbazole (AEC) solution (Dako, catalog K3464). Virus titer was recorded as PFU/gm of lung tissue.

Lung cell isolation and analysis. The lungs were removed and digested using collagenase $(0.7 \mathrm{mg} / \mathrm{ml})$ (Roche, catalog 10103578001) and DNase I (30 $\mu \mathrm{g} / \mathrm{ml}$ ) (Roche, catalog 10104159001) in RPMI-1640 medium (Gibco, catalog 61870-036) for 30 minutes at $37^{\circ} \mathrm{C}$. Lungs were dissociated on a gentleMACS Dissociator (Miltenyi Biotech) according to the manufacturer's protocol. Single-cell suspensions were obtained by passing the dissociated tissue through a 70- $\mu \mathrm{m}$ cell strainer (Fisher Scientific, catalog 22363548). RBC lysis was performed using BD Pharmlyse (BD Biosciences, catalog 555899). Cells were then counted and treated with PMA (50 ng/ml) (Sigma-Aldrich, catalog P8139), ionomycin (500 ng/ml) (Sigma-Aldrich, catalog I3909), and GolgiStop containing monensin (BD Biosciences, catalog 554724) for 4 hours. Cells were fixed with $2 \%$ paraformaldehyde and stained with fluorochrome-conjugated antibodies listed below.

Flow cytometry. The fluorochrome-conjugated antibodies used were anti-mouse CD3\&-PE-CF594 (BD Biosciences, catalog 562332, clone 145-2C11), CD8-PerCP/Cy5.5 (BD Biosciences, catalog 561109, clone 53-6.7),

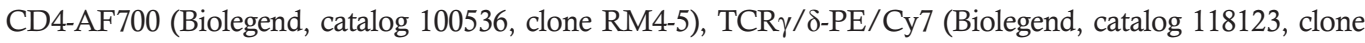
GL3), NK1.1-PerCP/Cy5.5 (Biolegend, catalog 108727, clone PK136), CD45-PerCP/Cy5.5 (Biolegend, cata$\log 103131$, clone 30-F11), and CD90.2 (Thy-1.2)-PE/Cy7 (Biolegend, catalog 140309, clone 53-2.1). $\gamma \delta \mathrm{T}$ cells and NK cells were identified by gating on $\mathrm{CD}^{+} \gamma \delta \mathrm{TCR}^{+}$cells and CD3-NK1.1 $1^{+}$cells, respectively. The lineage cell population was identified by staining with an APC-conjugated antibody cocktail containing TER-119 (Biolegend, catalog 116211, clone TER-119), CD5 (Biolegend, catalog 100625, clone 53-7.3), NK1.1 (Biolegend, catalog 108709, clone PK136), CD49b (Biolegend, catalog 108909, clone DX5), CD3 $\varepsilon$ (BD Biosciences, catalog 553066, clone 145-2C11), CD11b (BD Biosciences, catalog 553312, clone M1/70), CD11c (BD Biosciences, catalog 550261, clone HL3), CD45R/B220 (BD Biosciences, catalog 553092, clone RA3-6B2), Gr1/Ly-6GLy-6C (BD Biosciences, catalog 553129, clone RB6-8C5), and F4/80 (Invitrogen, catalog MF48005, clone BM8). ILC2 cells were then identified by gating on $\mathrm{Lin}^{-} \mathrm{CD} 45^{+} \mathrm{CD} 90.2^{+}$cells. Intracellular staining for cytokines was carried out using fluorophore-conjugated antibodies, which included anti-IL-17A-V450 (BD Biosciences, catalog 560522, clone TC11-18H10), anti-IFN- $\gamma$-APC (BD Biosciences, catalog 562018, clone XMG1.2), and anti-IL-13-PE (eBioscience, catalog 12-7133-81, clone eBio13A), following the manufacturers' instructions. To assess cell viability, the viability dyes used were FVD EFluor 780 (eBioscience, catalog 65-0865-14) and Ghost Dye Violet 510 (Tonbo Biosciences, catalog 13-0870). Stained cells were examined on BD LSR II flow cytometer (BD Immunocytometry Systems), and the data were analyzed using FlowJo software (TreeStar).

$R N A$ isolation and quantitative real-time PCR. Lung homogenates were treated with Qiazol lysis reagent (Qiagen, catalog 79306). RNA was isolated using the miRNeasy mini kit (Qiagen, catalog 217004) and treated with RNase-free DNase (Qiagen, catalog 79254) according to manufacturer's instructions. cDNA was synthesized using a High-Capacity cDNA Reverse Transcription Kit (Applied Biosystems, catalog 4368813) according to the manufacturer's instructions. Quantitative real-time PCR was performed using validated TaqMan Gene expression primer and probe sets (Life Technologies) for the following genes: Muc5ac (catalog Mm01276718_m1), Gob5 (catalog Mm01320697_m1), Il1ß (catalog Mm00434228_m1), Ifit1 (catalog Mm00515153_m1), Il6 (catalog Mm00446190_m1), Il23p19 (catalog Mm00518984_m1), Oasl2 (catalog Mm00496187_m1), RSV-L pol (customized catalog 5507366), and Hprt1 (catalog Mm03024075_m1). $R S V$ - $L$ polymerase gene expression was detected using the following primers and probe: forward primer (5'-GAACTCAGTGTAGGTAGAATGTTTGCA-3'), reverse primer (5'-TTCAGCTATCATTTTCTCTGCCAAT-3'), and probe (5'-TTTGAACCTGTCTGAACATTCCCGGTT-3'). All TaqMan primers were purchased from Life Technologies. Gene expression was calculated using the $2^{-\Delta \mathrm{Ct}}$ method (normalized to hprt1). Results were analyzed using SDS 2.2.2 software. 
ELISA. Lung homogenates were supplemented with 0.02\% Tween 20 (Sigma-Aldrich, catalog P2287) and Complete Mini, EDTA-Free Protease Inhibitor (Roche, catalog 04693159001). Debris-free supernatant was used for cytokine measurement. Levels of IL-1 $\beta$ protein in mouse lungs were measured using the Mouse DuoSet ELISA Kit (R\&D Systems, catalog DY401) according to the manufacturer's protocol. Culture supernatants from THP-1 cells were used to measure the level of IL-1 $\beta$ protein using the Human DuoSet ELISA Kit (R\&D Systems, catalog DY201).

Histological analysis. Fixed right lobes of the lungs were embedded in paraffin blocks, and $4-\mu \mathrm{m}$ sections were prepared for histological analysis. The sections were dried horizontally on a warming tray for 1 hour at $37^{\circ} \mathrm{C}$ and then stained with periodic acid-Schiff (PAS) or subjected to immunohistochemical staining.

PAS staining. PAS staining was performed to evaluate airway mucus and goblet cell hyperplasia. Lung sections were cleared from paraffin, rehydrated, and then stained with $0.5 \%$ Periodic acid (Sigma-Aldrich, catalog 375810) and Schiff's reagent (Rowley Biochemical, catalog SO-429) for airway mucus staining and with Mayer's hematoxylin (Sigma-Aldrich, catalog MHS32) for identifying nuclei. Mucus-producing goblet cells were semiquantitatively scored on a scale of 0 to $4(0,<5 \%$ mucus-producing goblet cells; 1 , $5 \%-25 \% ; 2,25 \%-50 \% ; 3,50 \%-75 \% ; 4,>75 \%$ ). Images were taken at $\times 10$ magnification.

Immunohistochemical IHC staining for neutrophils. After deparaffinization and rehydration, lung sections were blocked with 10\% goat serum (Jackson Immunoresearch, catalog 005-000-121) and stained overnight at $4^{\circ} \mathrm{C}$ with anti-neutrophil antibody (Abcam, catalog ab2557, clone NIMP-R14) in a humidified chamber. Tissue sections were then treated with $3 \% \mathrm{H}_{2} \mathrm{O}_{2}$ in TBS to quench endogenous peroxidase for 15 minutes, followed by HRP-conjugated goat anti-rat IgG (Jackson Immunoresearch, catalog 112035-143) secondary antibody for 1 hour at room temperature. Positive cells (neutrophils) were visualized by treatment with diaminobenzidine substrate (DAB) (Abcam, catalog ab103723). Sections were counterstained with hematoxylin (Leica, catalog 3801571), dehydrated, and mounted. Images were obtained using a light microscope at a magnification of $\times 20$. Quantitation was performed using Nikon Elements, where thresholds were defined based on hue, saturation, and intensity to standardize the counting of individual nuclei and DAB-positive-stained neutrophils. 4-5 independent fields in the lung tissue section of each mouse were examined for quantitation, and the mean value obtained from each mouse was used for statistical analysis.

Immunofluorescent staining for RSV. Mouse lungs were fixed by inflating with 2\% PFA for 2-4 hours at $4^{\circ} \mathrm{C}$, and then the tissues were placed in $30 \%$ sucrose for 24 hours. 2-methyl butane and liquid nitrogen were used to freeze the tissues, and $7-\mu \mathrm{m}$ cryostat sections were prepared. After rehydration and permeabilization, lung sections were blocked using 5\% donkey serum (Jackson Immunoresearch, catalog 017-000-121) and stained overnight at $4^{\circ} \mathrm{C}$ in the dark with goat anti-RSV all antigen antibody (Meridian Life Science, catalog B65860G). The sections were then stained with Cy3-conjugated donkey anti-goat IgG (Jackson Immunoresearch, catalog 705-165-147) secondary antibody and F-actin counterstain AF488 phalloidin (Molecular Probes, catalog A12379) for 1 hour at room temperature in the dark. Nuclei were stained with Hoechst 33342 (Molecular Probes, catalog H3570) and sections were mounted with Gelvatol. Images were obtained on Nikon 90i scanning microscope under identical conditions at $\times 10$ magnification. Quantification was performed by assessing the percentage of RSV-infected tissue from total tissue using Nikon Elements software. A consistent, intensity-based threshold was used to generate a binary mask, indicating positive signal. 4-5 independent fields in the lung tissue section of each mouse were examined for quantitation, and the mean value obtained from each mouse was used for statistical analysis.

Statistics. The Student's unpaired 2-tailed $t$ test, 1-way ANOVA with the Tukey post-hoc test, the Mann-Whitney $U$ test, and the Kruskal-Wallis test were used as appropriate. Differences between groups were considered significant at $P<0.05$. All statistical analyses were performed using GraphPad Prism 7 software.

Study approval. All protocols related to the animal experiments, including housing and care, were approved by the institutional animal care and use committee for the University of Pittsburgh.

\section{Author contributions}

PR conceived the study. SD, MR, PR, and AR designed the experiments and analyzed the data. SD, MR, JC, TBO, and RH performed experiments and analyzed data. JVW helped in data analysis. SD, PR, and AR wrote the manuscript. 


\section{Acknowledgments}

This work was supported by National Institutes of Health grants AI100012, HL122307, and HL114453 (to PR) and HL113956, AI106684, and AI048927 (to AR). The authors thank S. Nouraie for assistance with statistical analysis of data and M. Jessup and M. Joseph for assistance with quantitation of imaging data.

Address correspondence to: Prabir Ray or Anuradha Ray, 3459 Fifth Avenue, NW628 MUH, Pittsburgh, Pennsylvania 15213, USA. Phone: 412.802.3192; Email: rayp@pitt.edu (P. Ray) or raya@pitt.edu (A. Ray).

1. Hall CB. Respiratory syncytial virus and parainfluenza virus. N Engl J Med. 2001;344(25):1917-1928.

2. Falsey AR, Hennessey PA, Formica MA, Cox C, Walsh EE. Respiratory syncytial virus infection in elderly and high-risk adults. N Engl J Med. 2005;352(17):1749-1759.

3. Johnson JE, Gonzales RA, Olson SJ, Wright PF, Graham BS. The histopathology of fatal untreated human respiratory syncytial virus infection. Mod Pathol. 2007;20(1):108-119.

4. Collins PL, Graham BS. Viral and host factors in human respiratory syncytial virus pathogenesis. $J$ Virol. 2008;82(5):2040-2055

5. Nair H, et al. Global burden of acute lower respiratory infections due to respiratory syncytial virus in young children: a systematic review and meta-analysis. Lancet. 2010;375(9725):1545-1555.

6. Openshaw PJM, Chiu C, Culley FJ, Johansson C. Protective and Harmful Immunity to RSV Infection. Annu Rev Immunol. 2017;35:501-532.

7. PrabhuDas $\mathrm{M}$, et al. Challenges in infant immunity: implications for responses to infection and vaccines. Nat Immunol. 2011;12(3):189-194.

8. Abarca K, et al. Safety, tolerability, pharmacokinetics, and immunogenicity of motavizumab, a humanized, enhanced-potency monoclonal antibody for the prevention of respiratory syncytial virus infection in at-risk children. Pediatr Infect Dis $J$ 2009;28(4):267-272.

9. Smart KA, Paes BA, Lanctôt KL. Changing costs and the impact on RSV prophylaxis. J Med Econ. 2010;13(4):705-708.

10. Moore EC, Barber J, Tripp RA. Respiratory syncytial virus (RSV) attachment and nonstructural proteins modify the type I interferon response associated with suppressor of cytokine signaling (SOCS) proteins and IFN-stimulated gene-15 (ISG15). Virol J. 2008;5:116.

11. Karron RA, et al. Respiratory syncytial virus (RSV) SH and G proteins are not essential for viral replication in vitro: clinical evaluation and molecular characterization of a cold-passaged, attenuated RSV subgroup B mutant. Proc Natl Acad Sci USA. 1997;94(25):13961-13966.

12. Teng MN, Whitehead SS, Collins PL. Contribution of the respiratory syncytial virus G glycoprotein and its secreted and membrane-bound forms to virus replication in vitro and in vivo. Virology. 2001;289(2):283-296.

13. Boyoglu-Barnum S, et al. A respiratory syncytial virus (RSV) anti-G protein $F\left(a b^{\prime}\right) 2$ monoclonal antibody suppresses mucous production and breathing effort in RSV rA2-line19F-infected BALB/c mice. J Virol. 2013;87(20):10955-10967.

14. Haynes LM, et al. Therapeutic monoclonal antibody treatment targeting respiratory syncytial virus (RSV) G protein mediates viral clearance and reduces the pathogenesis of RSV infection in BALB/c mice. J Infect Dis. 2009;200(3):439-447.

15. Tripp RA, Jones LP, Haynes LM, Zheng H, Murphy PM, Anderson LJ. CX3C chemokine mimicry by respiratory syncytial virus G glycoprotein. Nat Immunol. 2001;2(8):732-738.

16. White GE, Greaves DR. Fractalkine: one chemokine, many functions. Blood. 2009;113(4):767-768

17. Chirkova T, et al. CX3CR1 is an important surface molecule for respiratory syncytial virus infection in human airway epithelial cells. J Gen Virol. 2015;96(9):2543-2556.

18. Jeong KI, et al. CX3CR1 Is Expressed in differentiated human ciliated airway cells and co-localizes with respiratory syncytial virus on cilia in a g protein-dependent manner. PLoS ONE. 2015;10(6):e0130517.

19. Johnson SM, et al. Respiratory syncytial virus uses CX3CR1 as a receptor on primary human airway epithelial cultures. PLoS Pathog. 2015;11(12):e1005318.

20. Hallak LK, Collins PL, Knudson W, Peeples ME. Iduronic acid-containing glycosaminoglycans on target cells are required for efficient respiratory syncytial virus infection. Virology. 2000;271(2):264-275.

21. Hallak LK, Spillmann D, Collins PL, Peeples ME. Glycosaminoglycan sulfation requirements for respiratory syncytial virus infection. J Virol. 2000;74(22):10508-10513.

22. Malhotra R, et al. Isolation and characterisation of potential respiratory syncytial virus receptor(s) on epithelial cells. Microbes Infect. 2003;5(2):123-133.

23. Amanatidou V, Sourvinos G, Apostolakis S, Tsilimigaki A, Spandidos DA. T280M variation of the CX3C receptor gene is associated with increased risk for severe respiratory syncytial virus bronchiolitis. Pediatr Infect Dis J. 2006;25(5):410-414

24. Faure S, et al. Rapid progression to AIDS in HIV+ individuals with a structural variant of the chemokine receptor CX3CR1. Science. 2000;287(5461):2274-2277.

25. Jung S, et al. Analysis of fractalkine receptor CX(3)CR1 function by targeted deletion and green fluorescent protein reporter gene insertion. Mol Cell Biol. 2000;20(11):4106-4114.

26. Niess JH, et al. CX3CR1-mediated dendritic cell access to the intestinal lumen and bacterial clearance. Science. 2005;307(5707):254-258.

27. Everard ML, et al. Analysis of cells obtained by bronchial lavage of infants with respiratory syncytial virus infection. Arch Dis Child. 1994;71(5):428-432.

28. Stokes KL, et al. The respiratory syncytial virus fusion protein and neutrophils mediate the airway mucin response to pathogenic respiratory syncytial virus infection. J Virol. 2013;87(18):10070-10082.

29. Krishnamoorthy N, et al. Early infection with respiratory syncytial virus impairs regulatory $\mathrm{T}$ cell function and increases sus- 
ceptibility to allergic asthma. Nat Med. 2012;18(10):1525-1530.

30. Cua DJ, Tato CM. Innate IL-17-producing cells: the sentinels of the immune system. Nat Rev Immunol. 2010;10(7):479-489.

31. Lukacs NW, et al. Differential immune responses and pulmonary pathophysiology are induced by two different strains of respiratory syncytial virus. Am J Pathol. 2006;169(3):977-986.

32. Stokes KL, et al. Differential pathogenesis of respiratory syncytial virus clinical isolates in BALB/c mice. $J$ Virol. 2011;85(12):5782-5793.

33. Moore ML, et al. A chimeric A2 strain of respiratory syncytial virus (RSV) with the fusion protein of RSV strain line 19 exhibits enhanced viral load, mucus, and airway dysfunction. J Virol. 2009;83(9):4185-4194.

34. Lukacs NW, Smit JJ, Mukherjee S, Morris SB, Nunez G, Lindell DM. Respiratory virus-induced TLR7 activation controls IL-17-associated increased mucus via IL-23 regulation. J Immunol. 2010;185(4):2231-2239.

35. Johnson $\mathrm{CH}$, et al. Effect of chemokine receptor CX3CR1 deficiency in a murine model of respiratory syncytial virus infection. Comp Med. 2012;62(1):14-20.

36. Poccia F, Agrati C, Martini F, Capobianchi MR, Wallace M, Malkovsky M. Antiviral reactivities of gammadelta T cells. Microbes Infect. 2005;7(3):518-528.

37. Vantourout P, Hayday A. Six-of-the-best: unique contributions of $\gamma \delta$ T cells to immunology. Nat Rev Immunol. 2013;13(2):88-100.

38. Ribot JC, et al. Cutting edge: adaptive versus innate receptor signals selectively control the pool sizes of murine IFN- $\gamma$ - or IL-17-producing $\gamma \delta$ T cells upon infection. J Immunol. 2010;185(11):6421-6425.

39. Starner TD, Barker CK, Jia HP, Kang Y, McCray PB. CCL20 is an inducible product of human airway epithelia with innate immune properties. Am J Respir Cell Mol Biol. 2003;29(5):627-633.

40. Hammerich L, et al. Chemokine receptor CCR6-dependent accumulation of $\gamma \delta \mathrm{T}$ cells in injured liver restricts hepatic inflammation and fibrosis. Hepatology. 2014;59(2):630-642.

41. Kallal LE, Schaller MA, Lindell DM, Lira SA, Lukacs NW. CCL20/CCR6 blockade enhances immunity to RSV by impairing recruitment of DC. Eur J Immunol. 2010;40(4):1042-1052.

42. Fei M, et al. TNF-alpha from inflammatory dendritic cells (DCs) regulates lung IL-17A/IL-5 levels and neutrophilia versus eosinophilia during persistent fungal infection. Proc Natl Acad Sci USA. 2011;108(13):5360-5365.

43. Goritzka M, et al. Alveolar macrophage-derived type I interferons orchestrate innate immunity to RSV through recruitment of antiviral monocytes. J Exp Med. 2015;212(5):699-714.

44. Netea MG, Simon A, van de Veerdonk F, Kullberg BJ, Van der Meer JW, Joosten LA. IL-1beta processing in host defense: beyond the inflammasomes. PLoS Pathog. 2010;6(2):e1000661.

45. Hu SJ, et al. Upregulation of P2RX7 in Cx3cr1-deficient mononuclear phagocytes leads to increased interleukin-1 $\beta$ secretion and photoreceptor neurodegeneration. J Neurosci. 2015;35(18):6987-6996.

46. Dodd J, Riffault S, Kodituwakku JS, Hayday AC, Openshaw PJ. Pulmonary V gamma 4+ gamma delta T cells have proinflammatory and antiviral effects in viral lung disease. J Immunol. 2009;182(2):1174-1181.

47. Huang H, Saravia J, You D, Shaw AJ, Cormier SA. Impaired gamma delta T cell-derived IL-17A and inflammasome activation during early respiratory syncytial virus infection in infants. Immunol Cell Biol. 2015;93(2):126-135.

48. Yamaguchi Y, Harker JA, Wang B, Openshaw PJ, Tregoning JS, Culley FJ. Preexposure to CpG protects against the delayed effects of neonatal respiratory syncytial virus infection. J Virol. 2012;86(19):10456-10461.

49. Caballero MT, et al. TLR4 genotype and environmental LPS mediate RSV bronchiolitis through Th2 polarization. J Clin Invest. 2015;125(2):571-582.

50. Zhivaki D, et al. Respiratory syncytial virus infects regulatory B cells in human neonates via chemokine receptor CX3CR1 and promotes lung disease severity. Immunity. 2017;46(2):301-314.

51. Moore ML, et al. Cutting Edge: Oseltamivir decreases T cell GM1 expression and inhibits clearance of respiratory syncytial virus: potential role of endogenous sialidase in antiviral immunity. J Immunol. 2007;178(5):2651-2654.

52. Schickli JH, et al. Palivizumab epitope-displaying virus-like particles protect rodents from RSV challenge. J Clin Invest. 2015;125(4):1637-1647. 